\title{
Laser evoked potential: Analysis of the methodology in post herpetic neuralgia
}

\author{
Alessandro Caminiti ${ }^{1 *}$, Amelia Zaccaro ${ }^{1}$, Andrea Truini ${ }^{2}$, William Raffaeli ${ }^{1}$, Domenico De Grandis ${ }^{3}$ \\ ${ }^{1}$ Department of Palliative Care and Pain Therapy, Infermi Hospital, Rimini, Italy \\ ${ }^{2}$ Department of Neurological Science, La Sapienza University, Rome, Italy \\ ${ }^{3}$ Italian Clinical Neurophysiology Society, Rome, Italy \\ Email: ${ }^{*}$ alessandro.caminiti@gmail.com
}

Received 16 September 2011; revised 24 October 2011; accepted 8 November 2011

\begin{abstract}
Background: Selective activation and the contemporary recording of $A$ delta and $C$ fibers from Yap laser on the scalp is a new neurophysiological assessment, allowing the selective activation of the nociceptive system. Objectives: To evaluate if the damage of the nociceptive system was related to post herptic nevralgia (PHN) development in a sample of 26 patients affected by Herpes zooster (HZ); secondly to assess if the impairment of thermal pathway and PHN development were related. Methods: Thirty-two patients were selected for the study, 26 of these were included in the study, whereas 6 were excluded because of cognitive impairment. All 26 study patients were submitted to LEP analysis at baseline (T0) and after six months (T1), and the correlation between clinical thermal disease and the development of PHN was monitored. Results: Pain duration was evidenced by the presence or absence of an instrumental signal in patients with acute $\mathrm{HZ}$ infection. There was total concordance between the absence of LEP signal and pain duration. The concordance between the clinical thermal disease and the pain duration was statistically significant for $43 \%$ of the sample. Conclusions: Our results suggested a possible role of LEP for PHN prognosis estimation; indeed, most patients affected by acute HZ, with absence of instrumental LEP signal had pain $>6$ months. We also noted a significant $(43 \%$ of cases) clinical concordance between the thermal pathway damage, the absence of instrumental signal and PHN development. Further studies are needed to address this issue.
\end{abstract}

Keywords: LEP; A Delta Fibers; C-Fibers; PHN Development; Pain; Spino-Thalamic Pathway

"Corresponding author.

\section{INTRODUCTION}

Event-related brain potential provides significant information about sensory and cognitive processes which may occur in the brain. The cortical processes characterizing pain are highly behind of other sensory modalities; the difficulties related to the production of an adequate and selective nociceptive stimulus most probably contributes to this event.

The introduction of laser stimulus allows the selective activation of the nociceptive system, activating predominantly A delta and C fiber nociceptors (Iannetti et al., 2006) [1]. Concurrent activation of the sensory modalities, such as A beta fibers of the lemniscal pathway, should be avoided, as their activation could produce overlapping responses and, more importantly, modulate themselves nociceptive responses.

Monochromatic radiant heat sources of high power density, such as infrared or YAP lasers, are able to produce stimuli whose spectral energy density is several orders of magnitude greater than those produced by conventional light sources (Bromm and Treede, 1987) [2]. Studies on cutaneous receptors, furthermore, show that laser stimulation allows the selective activation of $\mathrm{A}$ delta and $\mathrm{C}$ fibers nociceptors (Plaghki and Mouraux, 2003) [3]. It is known that somatosensory system, signaling temperature and pain perception, uses a different set of afferent and central pathways, whose function is accessible by laser evoked potential (Treede et al., 2003 and 1988) $[4,5]$. For this reason, the Laser Evoked Potential (LEP) can document lesions of the spinal-thalamic tract, lateral brain system, and thalamic-cortical projections, transporting thermal nociceptive signals. Many clinical studies recently show that LEP can be useful for establishing clinical diagnosis, when deficit of nociceptive system are present (Cruccu and Garcia-Larrea, 2004) [6] (Mouraux, 2005) [7].

Herpes Zoster infection is a frequent pathology caused by Varicella Zoster virus, which is usually located in 
para spinal and in trigeminal ganglions. International literature reports an increase of illness incidence in relation to two main factors: age, with a maximum peak after 60 years old, and presence of neoplastic or immune-system pathologies. Clear prognostic factors are still missing to estimate the probably of chronic pain development for the each patient during the acute phase of infection. PHN is a frequent clinical condition that is difficult to treat. Intratecal drugs administrations, or neuromodulation therapy are often employed for pain control, even if they do not give good results in many cases, because the pain typology is central. Infection from Varicella Zoster (HZ) virus represents a good model for studying neurophysiological mechanism responsible of neuropathic pain onset (Wall, 1993; Watson, 1988) [8]. LEPs are considered the best tool for a delta pathway dysfunction assessment, as suggested by the European Federation of the Neurological Societies (EFNS) guidelines on neuropathic pain assessment (Cruccu, G. et al., 2010) [9]. For these reasons, in our study, we examined $\mathrm{HZ}$ acute patients using LEP for the evaluation of LEP ability in pain prognosis.

In this preliminary study we monitored 26 patients, affected by acute HZ, with LEP analysis, in order to assess if the complete or partial damage of the spino-thalamic pathway could be an index for pain persistence, and if the damage of the nociceptive system was strictly related to pain duration.

\section{MATERIALS AND METHODS STUDY DESIGN}

This is an open label prospective observational study, which was performed at the Pain and Palliative Care Unit of the Infermi Hospital of Rimini, Italy.

\subsection{Ethics}

The study was approved by the Hospital Ethics Committee and conducted according to the Helsinki declaration principles on human clinical studies. All the patients, after a complete explanation of the procedure and the aims of the study, gave a written consent.

\subsection{The Sample}

Thirty-two consecutive patients affected by Varicella Zoster, and suffering from pain were selected for the study; 26 of them were included in the study, whereas 6 were excluded because of cognitive impairment. We included in the study only patients in the same stage of disease, within maximum 7 days after pathology onset, and responding to the following inclusion criteria, $>18$ years old, informed consent signed, pain intensity, measured with the Visual Analogue Scale (VAS), diagnosis of
PHN or Varicella Zoster infection, Varicella Zoster infection for the acute phase group. Exclusion criteria: body mass index $(\mathrm{BMI})>25$, cognitive impairment.

\subsection{Interventions}

At baseline visit (T0) neurological and instrumental examinations were performed and BMI was computed. Neurophysiological examination was repeated after six months (T1). Sensory disturbances were carefully assessed. Patients were examined for negative (tactile, pinprick, and thermal hypoesthesia) and positive symptoms (Constant pain, paroxysmal pain, itching, mechanical and cold allodynia and pinprick hyperalgesia). Thermal examination was evaluated with a test tube previously heated at $32^{\circ} \mathrm{C}$ (which is the mean for heat sensibility in normal subjects) and at $28^{\circ} \mathrm{C}$ (mean cold sensitivity) (Rowbotham and Fields, 1989) [10].

The following parameters were also registered at the baseline visit: concomitant pathologies, such as neoplastic or immunodeficiency pathologies; age (since PHN development is age dependent, with a maximum peak of incidence after 60 years) (Hempenstall et al., 2004) [11]; concomitant therapy, such as anti-epileptics, antidepressants, clonidine, baclofen; pain therapy; pain intensity, measured with VAS 0-10; and pain duration.

At the baseline visit, each patient received the same treatment: oral or, where possible, an intratecal (lumbar, dorsal) therapy, with peripheral nerves anaesthetic or ganglion blocks in the cranial district. The neurophisyological test was performed in the same position of the block, but generally two or three days after the anesthetic block, not to influence or interfere with the data.

\section{INSTRUMENTAL EXAM}

We applied a cranial standard setting with 6 electrodes, in order to record LEP, as follows: CZ-forehead, T3-FZ, T4 FZ Electrodes recorded eye movement. Laser stimuli were applied at skin level, by laser Yag, with the following parameters for A delta fibres: diameter of the spot 5 $\mathrm{mm}$; scanning time $2 \mathrm{~m} / \mathrm{sec}$; power range of stimulus $0.50 \mathrm{~J}-2 \mathrm{~J}$; for $\mathrm{C}$ fibres: diameter of the spot $15 \mathrm{~mm}$, scanning time $10 \mathrm{~m} / \mathrm{sec}$, power range of stimulus $3 \mathrm{~J}$ $10.25 \mathrm{~J}$.

According to recent debates in literature about the poor reproduction and reliability of $C$ fibers recording in LEP, we decided not to study these fibers in upper and lower limbs and in the chest, whereas they were included in the cranial district.

Once the patient was adequately relaxed, we performed: a threshold evaluation of A delta fibers stimuli perception in limbs and chest, and of A delta and C fibers in the trigeminal territory; and a nociceptive recording potential threshold evaluation. 
The stimulus was supplied at irregular intervals, in order to avoid the neurophysiological phenomenon called habituation that could compromise the signals recording. For this reason we performed 3 runs of 10 irregular stimuli, the electromyograph gave the average of the 10 simuli for each run as output, and the signal average of the three runs was compute for each patient.

The signal was considered absent when LEP $=0$, whereas the cut off of LEP values were related to standard values (see reference Truini et al., 2005) [12].

Signal curves were evaluated taking into account sex, age and height values (Traini et al., 2005) [12]. We then classified LEP signals as follows: standard signal = normal, pathologic signal $=$ pathologic, absent signal $=$ absent.

\subsection{Parameters Monitored}

The following parameters were monitored during the study: presence or absence of instrumental signal and pain duration; modification of instrumental signal and clinical pain trend; potential correlation and corresponddence between clinical and instrumental examination of thermal sensitivity; improvement of clinical thermal sensitivity and its association with pain duration. We have carefully classified clinical cold and heat sensitivity.

\subsection{Outcome Measures}

LEP prediction of pain prognosis was considered positive if pain duration was $<6$ months for patients with the presence of LEP signal (normal or pathological), and months for those with absent LEP signal. We also assessed the clinical index between clinical thermal disease and the PHN development.

\subsection{Statistical Analysis}

Continuous data of the whole sample $(n=26)$ were re- ported as the mean and standard deviation, and were analyzed by the Mann-Whitney test, statistical significance was defined as $\mathrm{P}<0.05$. Proportions were expressed in percentage Table 2.

\section{RESULTS}

\subsection{The Sample}

We studied 26 patients in acute HZ infection phase (16 males +10 females), the average age of the sample was 68.69 years. 14 patients were affected by thoracic, 5 patients by trigeminal (vi) and 5 patients by upper limbs herpes. 13 patients did not have any other concomitant pathology, whereas 13 patients had the following concomitant pathologies: 4 patients affected by cancer, 2 patients by self immune pathology, 1 patient affected by epilepsy, 2 patients affected by diabetes, 1 patient affected by depression, 3 patients affected by chronic trigeminal neuralgia.

1 patient underwent radiotherapy treatment for prostate cancer, and 1 patient had chemotherapy. None of the patients examined underwent immunosoppressive treatment in the previous year. As concerning the concomitant therapy that could potentially interfere with pain trend (antidepressant, antiepileptic), 3 patients used pharmacological poly-therapy before the development of HZ.

\subsection{LEP Signal and Pain Duration}

15 patients with normal LEP signal had pain duration $<6$ months; whereas 11 patients with LEP signal absence had the pain duration $>6$ months $(p<0.001)$ (Figure 1).

\subsection{Clinical Thermal Sensitivity and Pain Duration}

The presence of clinical thermal disease was established

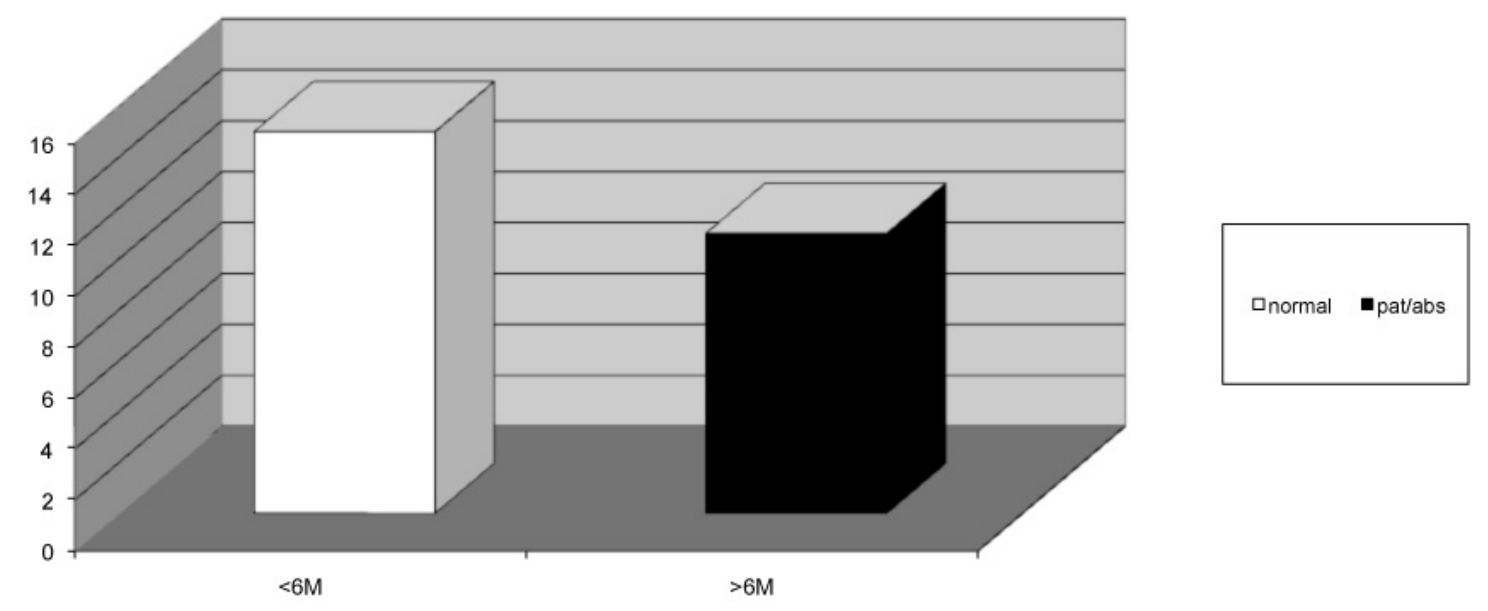

Figure 1. Correlation between pain duration and LEP signal. 
if there was at least of one clinical deficit of thermal pain (warm or cold hypoestesia). There was a positive concordance between the presence of clinical thermal sensitivity diseases, LEP absence and the development of chronic pain for 13 patients. We did not find any correlation between these factors for 6 patients. Patients with instrumental absent signal and clinical thermal disturbances were $57 \%$ of the entire group (Figure 2).

\section{DISCUSSION}

We know the value of the different factors involved in the development of PHN, such as age $>60$ years, immunodeficiency and cancer pathology (Jung et al., 2004) [13]; for this reason we carefully monitored the concomitant pathologies of our sample. The severity of the damage to the nociceptive system (Figure 1) was directly correlated with the persistence of pain. 15 patients with normal LEP signal, indeed, did not developed PHN, whereas 11 with the absence of LEP signal had persistent pain ( $>6$ months) (Figure 1). This result supported our initial hypothesis that the degree of nociceptive system damage was directly related to the development of PHN (Figure 2), indeed, there was a total concordance between the absence of signal and pain duration. Looking at Table 1, there were 2 patients (4 and 7) that showed LEP signal at T0, but not at T1. This result may be due to the fact that skin lesion does not enable epidermal receptors to transmit laser stimuli to A delta fibres in acute phases.

We also found the opposite clinical situation: absence of LEP signal at T0, followed by the appearance of the signal at T1. This may be due to an A delta fibers lesion, which can be found in every type of peripheral fibers lesion. In this case, the correspondent neurophysological findings appeared after 20 - 30 days, explaining the appearance of LEP signal in T1. For this reason we suggest to consider the entire cycle of LEP control in T0 and T1, and to repeat twice the LEP at T0 and T1; the main reason that we consider is to avoid false negative or positive; indeed, in the acute phase of inflammation epidermal receptors probably due to the edema, could temporary lack their capacity to transmit neurophysiological impulse. Another different situation could be constituted from the maintaining of the signal of compound action potential during the first 15 - 20 days after fiber lesion, as can be evidenced in peripheral fiber lesions.

The presence of signal at $\mathrm{T} 0$, but not at $\mathrm{T} 1$, in both patients number 4 and 7 , suggests that there may be a good prognostic concordance between LEP signal and the pain duration (Table 1). Another noteworthy aspect that we wanted to underline is that the mean age of the sample is quite high and this is one of the important risk factors found in literature for the development of PHN. The comparison of these two data (presence/absence of the signal and the age risk) enhances the importance of LEP signal presence in correlation to PHN development.

Only one of the patients with additional risk factors, such as immunodeficiency or neoplastic pathology, had LEP signal and did not develop chronic pain, whereas all the others patients without LEP signal did develop chronic pain. This result further supports our neur physiological hypothesis, as concerning neuropathic pain persistence; indeed, normal afferent impulse coming from peripheral nerves plays a protective and preventive role in the development of neuropathological circuits in peripheral and central nervous System (Woolf C-1993; Campbell) [14]. Clinical examination of thermal sensitivity was performed analyzing 6 clinical variables: heat hypostesia, cold hypoestesia, heat hyperestesia, cold hyperestesia, heat allodynia and cold allodynia. This analysis should confirm the utility of the clinical examination of heat and cold fibers in the development of PHN and also the concordance with instrumental data. Unfortunately there was not a statistical significance (57\%) (Figure 2). Our study has a great limitation because of sample size, which is small (26 patients), nonetheless, the results obtained are interesting and enlighten the potentiality of LEP in neuropathic pain development. Fur-

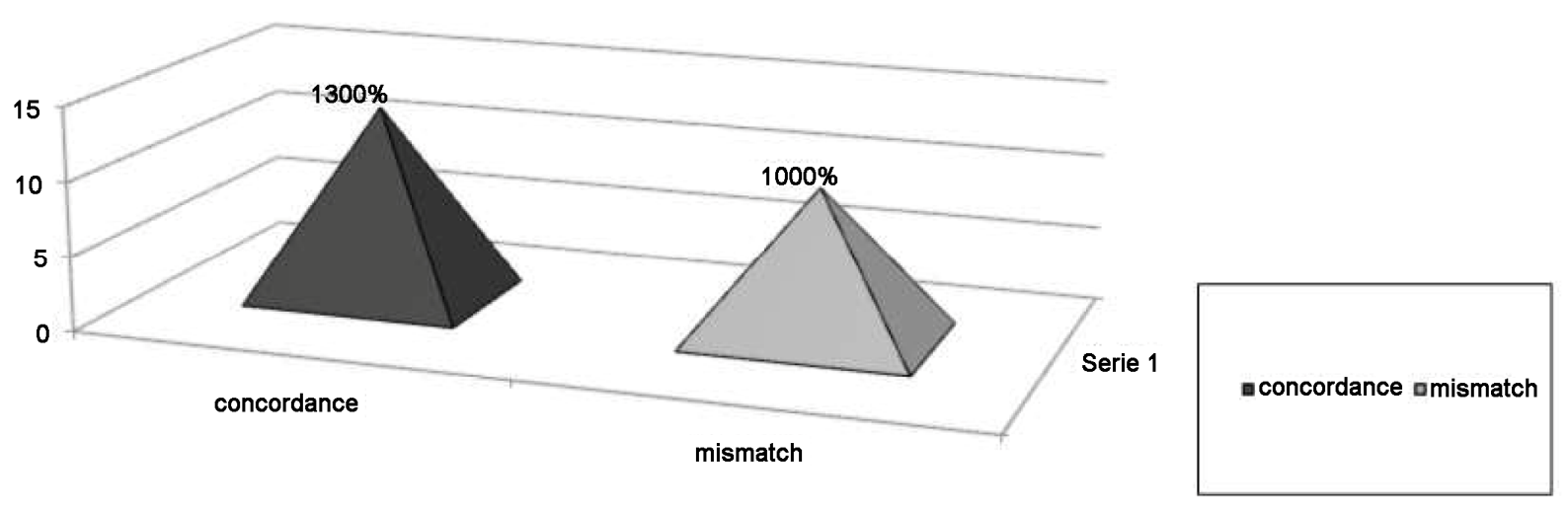

Figure 2. Correlation between thermal clinical impairment and absence of LEP signal. 
Table 1. Summarizing LEP table.

\begin{tabular}{|c|c|c|c|c|c|c|c|c|}
\hline pat & $\begin{array}{l}\text { NORMATIVE } \\
\text { VALUES }\end{array}$ & $\begin{array}{l}\text { AMP. } 1 \\
\text { microvolt }\end{array}$ & $\begin{array}{l}\text { LAT. } 1 \\
\text { millisec. }\end{array}$ & $\begin{array}{l}\text { AMP. } 2 \\
\text { microvolt }\end{array}$ & $\begin{array}{l}\text { LAT. } 2 \\
\text { millisec. }\end{array}$ & VAS 1 & VAS 2 & TOPOGRAPHY \\
\hline 1) & $190-270 \mathrm{msec}$ & $4.15 \underline{\mathrm{sx}}$ & $192 \underline{\mathrm{sx}}$ & Absent bilat. & Absent bilat. & 5 & 8 & D8-D9 dx \\
\hline 2) & $190-270 \mathrm{msec}$ & $13.3 \underline{\mathrm{dx}}$ & $270 \underline{\mathrm{dx}}$ & $13.3 \underline{\mathrm{dx}}$ & $13.3 \underline{\mathrm{dx}}$ & 5 & 5 & D9 sx. \\
\hline 3) & 190 - 270msec & $14.9 \underline{\mathrm{sx}}$ & $340 \underline{\mathrm{sx}}$ & $10 \mathrm{dx}$ & $280 \mathrm{dx}$ & 8 & 5 & D6-D8 dx. \\
\hline 4) & 130 - 200 msec & $11 \mathrm{dx}$ & $316 \mathrm{dx}$ & Absent bilat. & Absent bilat. & 9 & 6 & C2-C3 dx \\
\hline 5) & 130 - 200 msec & $20 \underline{\mathrm{dx}}$ & $210 \underline{\mathrm{dx}}$ & $25 \mathrm{sx}$ & $218 s x$ & 8 & 0 & V1 sx \\
\hline 6) & $170-270$ msec & $10 \mathrm{dx}$ & $170 \mathrm{dx}$ & $14.0 \mathrm{dx}$ & $200 \mathrm{dx}$ & 9 & 0 & D2-D4 dx \\
\hline 7) & 130 - 200 msec & $25 s x$ & $260 \mathrm{sx}$ & $24.8 \underline{\mathrm{dx}}$ & $181 \underline{\mathrm{dx}}$ & 8 & 6 & V1 sx \\
\hline 8) & $228-314 \mathrm{msec}$ & $18 \mathrm{dx}$ & $208 \mathrm{dx}$ & $20 \mathrm{dx}$ & $200 \mathrm{dx}$ & 1 & 0 & L5-S1 dx \\
\hline 9) & $190-270$ msec & $8.38 \mathrm{dx}$ & $216 \mathrm{dx}$ & $2.21 \mathrm{dx}$ & $213 d x$ & 9 & 0 & D10-D12 dx \\
\hline 10) & 130 - 200 msec & $10 \mathrm{sx}$ & 215 sx & $12.7 \mathrm{sx}$ & $221 s x$ & 8 & 0 & C6-C8 sx \\
\hline 11) & 190 - 270 msec & $15 \mathrm{sx}$ & 220 sx & $10.9 \mathrm{sx}$ & $222 \mathrm{sx}$ & 4 & 0 & D8-D10 sx \\
\hline 12) & 130 - 200 msec & $19.3 \underline{\mathrm{sx}}$ & $179 \underline{\mathrm{sx}}$ & $16.9 \mathrm{dx}$ & $314 d x$ & 9 & 5 & Plesso cerv.dx \\
\hline 13) & $130-200 \mathrm{msec}$ & $12.0 \mathrm{dx}$ & $205 \mathrm{dx}$ & $15.4 \mathrm{dx}$ & $214 d x$ & 9 & 0 & V3-C2 dx \\
\hline 14) & $190-270$ msec & $15 \underline{s \underline{x}}$ & $228 \underline{\mathrm{sx}}$ & $34.1 \mathrm{dx}$ & $223 \mathrm{dx}$ & 8 & 1 & D9-D10 dx \\
\hline 15) & $200-270$ msec & $36.6 \mathrm{sx}$ & $213 \mathrm{sx}$ & $30.8 \mathrm{sx}$ & $179 \mathrm{sx}$ & 3 & 0 & D11-D12 sx \\
\hline 16) & 190 - 270 msec & $20.5 \underline{\mathrm{dx}}$ & $224 \underline{\mathrm{dx}}$ & $6.84 \underline{\mathrm{dx}}$ & $336 \underline{\mathrm{dx}}$ & 7 & 9 & D8-D9 sx \\
\hline 17) & 190 - 270 msec & $8.55 \mathrm{dx}$ & $213 \mathrm{dx}$ & $9.00 \mathrm{dx}$ & $215 d x$ & $7-8$ & 0 & D8-D9 dx \\
\hline 18) & 190 - 270 msec & $6.96 \underline{\mathrm{sx}}$ & $245 \underline{\mathrm{sx}}$ & $12.8 \underline{\mathrm{sx}}$ & $234 \underline{s x}$ & 7 & 5 & D9-D10 dx \\
\hline 19) & $130-200 \mathrm{msec}$ & $21.9 \mathrm{sx}$ & $170 \mathrm{sx}$ & $26.1 \mathrm{sx}$ & $161 \mathrm{sx}$ & $4-5$ & 0 & Plesso cervicale+ Arnold sx \\
\hline 20) & 190 - 270 msec & $24 \mathrm{dx}$ & $199 \mathrm{dx}$ & $11.7 \mathrm{dx}$ & $204 d x$ & 7 & 0 & D8-D9 dx \\
\hline 21) & 190 - 270 msec & $17.5 \underline{\mathrm{dx}}$ & $228 \underline{\mathrm{dx}}$ & $43.5 \underline{\mathrm{dx}}$ & $266 \underline{\mathrm{dx}}$ & 7 & 7 & D8-D9 sx \\
\hline 22) & 130 - 200 msec & $15.7 \mathrm{sx}$ & $185 \mathrm{sx}$ & $16 \mathrm{sx}$ & $185 \mathrm{sx}$ & 6 & 0 & C3-C4 sx \\
\hline 23) & $130-200$ msec & $5.96 \mathrm{dx}$ & $195 \mathrm{dx}$ & $40 \mathrm{dx}$ & $182 \mathrm{dx}$ & 6 & 1 & $\mathrm{~V} 1 \mathrm{dx}$ \\
\hline 24) & 190 - 270 msec & $27.1 \underline{\mathrm{sx}}$ & $169 \underline{\mathrm{sX}}$ & Absent bilat. & Absent bilat. & 7 & 4 & D8 dx \\
\hline 25) & 130 - 200 msec & $60.8 \mathrm{sx}$ & $176 \mathrm{sx}$ & $60.8 \mathrm{sx}$ & $180 \mathrm{sx}$ & $8-9$ & 0 & V3 + plesso cervicale superficiale sx \\
\hline 26) & $130-200 \mathrm{msec}$ & $25 \underline{\mathrm{dx}}$ & $211 \underline{\mathrm{dx}}$ & $9.67 \underline{\mathrm{dx}}$ & $203 \underline{\mathrm{dx}}$ & 8 & 6 & V1 sx \\
\hline
\end{tabular}

Indicates that pathological side was not found; Patients with LEP at first control and absent LEP at the second control; LEP at the begining with signal recovery at second control.

Table 2. Difference VAS = VAS1 - VAS2 (positive value are improvements, while negative values are worsening).

\begin{tabular}{ccc}
\hline & $->$ lep $=0$ (ABSENT) & \\
\hline Obs & 11 & Largest 4 \\
Smallest & -3 & \\
Median & 2 & 1.272727 \\
Mean & \multicolumn{2}{c}{} \\
Std. Dev. & \multicolumn{2}{c}{ Largest 9 lep =1 (present) } \\
& 15 & \\
Obs & 1 & \\
Smallest & 7 & 6.433333 \\
Median & & 2.448518 \\
Mean & &
\end{tabular}

Mann-Whitney TEST was used to check if this difference in VAS improvement was significative (note median value in LEP 1 is 7 while median value in lep $=0$ is 2) probability $=0.0002$. ther studies are needed to better understanding the mechanisms of neuropathic pain.

\section{CONCLUSIONS}

According to EFNS guidelines we used LEP in acute phase of $\mathrm{HZ}$ for the assessment of neuropathic pain. Our results suggested that there might be a direct relationship between the damage of the specific nociceptive system and PHN development. The small sample size is a great limitation of our study and does not allow us to demonstrate the prognostic capacity of LEP in the development of PHN. Further studies are needed to address this issue.

Since this pathology is a paradigmatic model for the study of neuropathic pain neurophysiological mechanism, LEP examination could be used also to assess the prognostic capacity of invasive treatment applied to neuropathic pain. If our preliminary results will be confirmed in further studies, there could be important consequences in the timing of interventions and in the outcome of these 
pathologies.

\section{REFERENCES}

[1] Iannetti, G.D., Zambreanu, L. and Tracey, I. (2006) Similar nociceptive afferents mediate psychophysiological and electrophysiological responses to heat stimululation of glabrous and hairy skin in humans. The Journal of Physiology, 577, 235-248. doi:10.1113/jphysiol.2006.115675

[2] Bromm, B. and Treede, R.D. (1987) Pain related cerebral potential: Late and ultralate components. International Journal of Neuroscience, 33, 15-23. doi:10.3109/00207458708985926

[3] Plaghki, L. and Mouraux, A. (2003) How do we selectively activate skin nociceptors with a high power infrared laser? Physiology and bioptics of laser stimulation. Neurophysiologie Clinique/Clinical Neurophysiology, 33, 269-277. doi:10.1016/j.neucli.2003.10.003

[4] Treede, R.D., Lorenz J. and Baumagartner, U. (2003) Linical usefulness of laser evoked potentials. Neurophysiologie Clinique, 33, 303-314.

[5] Treede, R.D., Kief, S., Holzer, T. and Bromm, B. (1988) Late somatosensory evoked cerebral potential in response to cutaneous heat stimuli. Electroencephalography and Clinical Neurophysiology, 70, 429-441. doi:10.1016/0013-4694(88)90020-X

[6] Cruccu, G. and Garcia-Larrea, L. (2004) Clinical utility of pain-laser evoked potentials. Supplement to Clinical Neurophysiology, 57, 101-110. doi:10.1016/S1567-424X(09)70348-4

[7] Mouraux, A. (2005) Preceding events condition the central processing of nociceptive input as revealed by laserevoked potentials. Thesis, Laboratoire de Neurophysiologie (NEFY), Université Catholique de Louvain, OttigniesLouvain-la-Neuve.

[8] Wall, P.D. (1993) An essay on the mechanism which may contribute to the pain of posthereptic neuralgia. In: Watson, C.P.N., Ed., Herpes Zoster and Postherpetic Neuralgia, Elsevier Science Publishers B.V., Amsterdam, 121.

[9] Cruccu, G., Sommer, C. and Coli, P. (2010) European guide-lines for the assesment of neuropathic pain. European Journal of Neurology, 8 March.

[10] Rowbotham, M.C. and Fields, H.L. (1989) Post-herpetic neuralgia: The relation of pain compliant, sensory disturbance and skin temperature. Pain, 39, 129-144. doi:10.1016/0304-3959(89)90001-8

[11] Hempenstall, K., Nurmikko, T.J., Johnson, R.W., A’Hern R.P. and Rice, A.S. (2005) Analgesic therapy in postherpetic neuralgia: A quantitative systematic review, PLoS Medicine, 2, 0628-0644.

[12] Truini, A., Galeotti, F. Romaniello, A., Virtuoso M., Iannetti, G.D. and Cruccu, G. (2005) Laser-evoked potentials: Normative values. Clinical Neurophysiology, 116, 821-826.

[13] Jung, B.F., Johnson, R.W., Griffin, D.R. and Dworkin, R.H. (2004) Risk factors for postherpetic neuralgia in patients with herpes zoster. Neurology, 62, 1545-1551.

[14] Campbell, J.N., Raja, S.N., Mejers, R.A. and Mackinnon, S.E. (1988) Myelinated afferents signal the hyperalgesia associated with nerve injury. Pain, 32, 89-94. doi:10.1016/0304-3959(88)90027-9 